\title{
An Application of Theorems of Harish-Ghandra and Ado
}

\author{
Ryûki MATSUdA*
}

Let $G_{1}$ and $G_{2}$ be compact groups, $G_{1} *$ and $G_{2} *$ the sets of representations of $G_{1}$ aud $G_{2}$ respectively. Then the set of homomorphisms of $G_{1}$ into $G_{2}$ and the set of homomorphisms of $G_{*}^{*}$ into $G_{1} *$ are canonically homeomorphic to each other [4]. The similar results are also valid for locally compact groups [3].

Now let $g$ be a Lie algebra over an algebraically closed field $K$ of characteristic zero, $g^{*}$ be the set of representations of $g$. A map $\mu$ which assigns to every $D \in \mathrm{g}^{*}$ a matrix $\ell^{\prime}(D)$ of the same degree as $D$ is called a representation of $g^{*}$ it it preserves similarity, direct sum and Kronecker sum. The set $g^{* *}$ of representations of $g^{*}$ canonically becomes a Lie algebra (not necessarily finite dimensional). For $X \in \mathfrak{g}$ and $D \in$ $\mathfrak{g}^{*}$, we set $(\tau(X))(D)=D(X) . \quad \tau$ is a homomorphism of $\mathfrak{g}$ into $\mathrm{g}^{* *}$. If $\mathrm{g}$ is semisimple, the map $\tau$ is surjective [2]. On the other hand, as $g$ has a faithful representation (theorem of Ado, [1] ), we see that $\tau$ is a one-to-one mapping. Therefore $g$ and $g^{* *}$ are isomorphic to each other.

Let $g_{1}$ and $g_{2}$ be semi-simple Lie algebras over $K, g_{1}{ }^{*}$ and $g_{2} *$ be the sets of representations of $g_{1}$ and $g_{2}$ respectively. A map $\varphi$ of $g_{2}{ }^{*}$ into $g_{1} *$ is called a homomorphism if it preserves degree, similarity, direct sum and Kronecker sum. Let $F$ be the set of homomorphisms of $g_{1}$ into $g_{2}$, $\Phi$ the set of homomorphisms of $g_{2} *$ into $\mathfrak{g}_{2} *$. For $f=F$ and $D \in \mathrm{g}_{2}{ }^{*}$, we set $(\theta(f))(D)=D$ 。 $f$, where '。' is the composition of maps. Then the map $\theta(f)$ belongs to $\Phi$. And we have a map $\theta$ of $F$ into $\Phi$.

Let $\varphi$ be any element of $\Phi$. We have a homomorphism $\psi$ of $g_{1} * *$ into $\mathfrak{g}_{2}$ ** if we set $\phi(\mu)=\mu \circ \varphi$ for $\mu \in g_{1} * *$. Then $f=\tau_{2}{ }^{-1} \circ \psi \circ \tau_{1}$ is an element of $F$ and we have $\theta(f)=\varphi$, where $\tau_{1}$ and $\tau_{2}$ are the canonical isomorphisms of $\mathfrak{g}_{1}$ onto $\mathfrak{g}_{1}^{* *}$ and $\mathfrak{g}_{2}$ onto $\mathrm{g}_{2} * *$ respectively. Therefore the map $\theta$ is surjective.

Now if $f_{1}$ and $f_{2}$ are two distinct elements of $F$, we have $f_{1}(X) \neq f_{2}(X)$ for some $X \in g_{1}$ and $\tau_{2}\left(f_{1}(X)\right) \neq \tau_{2}\left(f_{2}(X)\right)$ and accordingly $D\left(f_{1}(X)\right) \neq D\left(f_{2}(X)\right)$ for some $D \in g_{2}{ }^{*}$. We have therefore $\theta\left(f_{1}\right) \neq \theta\left(f_{2}\right)$.

Tнвовем Let $K$ be an algebraically closed field of characteristic zero, g a semi-simple Lie algebra over $K, \mathfrak{g}^{* *}$ the representations of representations of $\mathfrak{g}$. Then $\mathfrak{g}$ and $\mathfrak{g}^{* *}$ are canonically isomorphic to each other.

*Department of Mathematics, Ibaraki University, Mito, Japan 
Let $\mathrm{g}_{1}$ and $\mathrm{g}_{2}$ be semi-simple Lie algebras over $K, \mathrm{~g}_{1}{ }^{*}$ and $\mathrm{g}_{2}{ }^{*}$ be the sets of representations of $\mathrm{g}_{1}$ and $\mathrm{g}_{2}$ respectively. Then the set $F$ of homomorphisms of $\mathfrak{g}_{1}$ into $\mathrm{g}_{2}$ and the set $\Phi$ of homomorphisms of $\mathfrak{g}_{2} *$ into $\mathfrak{g}_{1} *$ are canonically bijective to each other.

\section{References}

[1] C. Chevalley, Théorie des groupes de Lie, II, Paris (Hermann) (1955).

[2] HARISH-CHANDRA, Lie algebras and the Tannaka duality theorem, Annals of Math., 51 (1950), 299-330.

[3] R. MATSUDA, Notes on the duality theorem of locally compact groups, to appear on this Bulletin.

[4] K. SUZUKI, Notes on the duality theorem of non-commutative topological groups, Tohoku Math. Journ., 15 (1963), 182-186. 